\title{
Variation in Tibial Functionality and Fracture Susceptibility Among Healthy, Young Adults Arises From the Acquisition of Biologically Distinct Sets of Traits
}

\author{
Karl J Jepsen, ${ }^{1}$ Rachel Evans, ${ }^{2}$ Charles H Negus, ${ }^{3}$ Joel J Gagnier, ${ }^{1}$ Amanda Centi, ${ }^{2}$ Tomer Erlich, ${ }^{4}$ \\ Amir Hadid, ${ }^{4}$ Ran Yanovich, ${ }^{4}$ and Daniel S Moran ${ }^{4}$ \\ ${ }^{1}$ Department of Orthopaedic Surgery, The University of Michigan, Ann Arbor, MI, USA \\ ${ }^{2}$ U.S. Army Research Institute of Environmental Medicine, Natick, MA, USA \\ ${ }^{3}$ L-3 Jaycor, San Diego, CA, USA \\ ${ }^{4}$ Heller Institute, Sheba Medical Center, Ariel University, Israel
}

\begin{abstract}
Physiological systems like bone respond to many genetic and environmental factors by adjusting traits in a highly coordinated, compensatory manner to establish organ-level function. To be mechanically functional, a bone should be sufficiently stiff and strong to support physiological loads. Factors impairing this process are expected to compromise strength and increase fracture risk. We tested the hypotheses that individuals with reduced stiffness relative to body size will show an increased risk of fracturing and that reduced strength arises from the acquisition of biologically distinct sets of traits (ie, different combinations of morphological and tissue-level mechanical properties). We assessed tibial functionality retrospectively for 336 young adult women and men engaged in military training, and calculated robustness (total area/bone length), cortical area (Ct.Ar), and tissue-mineral density (TMD). These three traits explained 69\% to $72 \%$ of the variation in tibial stiffness $(p<0.0001)$. Having reduced stiffness relative to body size (body weight $\times$ bone length) was associated with odds ratios of 1.5 (95\% confidence interval $[\mathrm{Cl}], 0.5-4.3)$ and $7.0(95 \% \mathrm{Cl}, 2.0-25.1)$ for women and men, respectively, for developing a stress fracture based on radiography and scintigraphy. K-means cluster analysis was used to segregate men and women into subgroups based on robustness, Ct.Ar, and TMD adjusted for body size. Stiffness varied $37 \%$ to $42 \%$ among the clusters ( $p<0.0001$, ANOVA). For men, $78 \%$ of stress fracture cases segregated to three clusters ( $p<0.03$, chi-square). Clusters showing reduced function exhibited either slender tibias with the expected Ct.Ar and TMD relative to body size and robustness (ie, well-adapted bones) or robust tibias with reduced residuals for Ct.Ar or TMD relative to body size and robustness (ie, poorly adapted bones). Thus, we show there are multiple biomechanical and thus biological pathways leading to reduced function and increased fracture risk. Our results have important implications for developing personalized preventative diagnostics and treatments. ( 2013 American Society for Bone and Mineral Research.
\end{abstract}

KEY WORDS: FUNCTIONAL ADAPTATION; STRESS FRACTURES; BIOMECHANICS; TIBIA; ADULT

\section{Introduction}

$\mathrm{O}$ ne objective of the functional adaptation process is to ensure that a bone is mechanically functional, which means it is sufficiently stiff and strong to support the loads engendered during daily activities. Individuals that fracture exhibit different bone traits (ie, measures of morphology and tissue-level mechanical properties) compared to those that do not fracture. ${ }^{(1,2)}$ However, it remains unclear whether these trait variants indicate that the functional adaptation process was impaired and strength was compromised. We showed recently that the skeletal system coordinates several morphological and tissue-level mechanical properties relative to the natural variation in bone robustness (a measure of transverse size relative to length) in order to achieve mechanical functionality. ${ }^{(3-8)}$ Because an individual's bone function is defined by the particular combination of traits (ie, set of traits) acquired during growth and maintained during aging, it is difficult to presume impaired functionality based on variation in a single trait alone.

We hypothesize that the adaptive coordination among traits results in individuals having reduced strength for different biomechanical reasons. First, the natural variation in robustness

Received in original form August 23, 2012; revised form December 17, 2012; accepted December 31, 2012. Accepted manuscript online January 29, 2013. Address correspondence to: Karl J Jepsen, PhD, University of Michigan, Department of Orthopaedic Surgery, Room 2007, Biomedical Science Research Building, 109 Zina Pitcher Place, Ann Arbor, Ml 48109-2200. E-mail: kjepsen@umich.edu Additional Supporting Information may be found in the online version of this article.

Journal of Bone and Mineral Research, Vol. 28, No. 6, June 2013, pp 1290-1300 
is associated with functional inequivalence, at least in the tibia, whereby biological processes are unable to adjust traits such as cortical area and tissue-modulus to the degree needed to establish the same level of functionality among individuals; this resulted in slender tibias being two to three times less stiff relative to body size compared to robust tibias. ${ }^{\left({ }^{(8)}\right.}$ Functional inequivalence means that bone strength varies naturally among individuals, which may help explain why individuals with slender bones show an increased risk of fracturing throughout life..$^{(1,9-12)}$ Second, reduced functionality may also result from genetic and environmental factors that limit the degree to which traits such as cortical area or tissue-modulus are adjusted relative to robustness. ${ }^{(13)}$ This phenomenon has not been studied directly, but is supported by studies reporting that individuals with wide bones and a proportionally thin cortex show a higher fracture risk. ${ }^{(14,15)}$ The functional inequivalence combined with the variation in compensation superimposed on the variation in robustness together make it difficult to assume that all individuals have reduced functionality for the same biomechanical reason. To what extent the variation in functionality and fracture risk can be attributed to these two phenomenon remains unclear. Finding that individuals fracture for different biomechanical reasons will benefit efforts to reduce fracture risk because these individuals may require different prophylactic treatment strategies to strengthen bone.

The goal of this study was to determine how the natural variation in trait sets acquired by individuals relates to mechanical functionality. Variation in trait sets refers to variation in the combination of morphological and tissue-level mechanical properties acquired by individuals during growth. We tested the hypotheses that reduced tibial functionality (ie, stiffness relative to body size) can arise from the acquisition of biologically distinct sets of traits and that individuals with reduced tibial functionality will show an increased risk of fracturing. We tested these hypotheses by relating measures of tibial function with stress fracture incidence for individuals engaged in military training. Having reduced bone stiffness relative to body size may not be a problem for daily activities, given the large safety factors that allow the system to tolerate a modest degree of functional variation. ${ }^{(16)}$ However, reduced stiffness may lead to an increased risk of fracturing under extreme loading conditions, such as fatigue loads applied over a time frame that is too short to allow for a corrective adaptive response. Military training is an example of this condition, which involves young adults developing stress fractures despite being sufficiently healthy to pass rigorous medical exams.

\section{Subjects and Methods}

\section{Participants}

A total of 351 individuals (217 women, 134 men) volunteered from within the Israel Defense Forces to participate in this study, all with informed consent. The study was approved by the institutional review boards of the Committee for Research on Human Subjects, Israel Defense Forces (IDF), the Human Use Review Committee of the Sheba Medical Center, Tel Hashomer, Israel, and by the Human Use Review Committee of the Army Research Institute of Environmental Medicine, Natick, MA, USA.
The female cohort was engaged in light infantry training and the male cohort was engaged in elite combat training. The duration of training was 4 months. Of those enrolled, 336 individuals (204 women, 132 men) had valid information regarding anthropometric traits (body weight, tibial length) and morphological traits that were absent of motion artifacts leading to poor quality $\mathrm{PQCT}$ images.

\section{Bone morphology and tissue-mineral density}

Morphological traits of the tibial diaphysis were quantified from cross-sectional images generated using PQCT (XCT 2000; Stratec Medizintechnik, Pforzheim, Germany), as described. ${ }^{(17)}$ Measurement quality was assured daily using a standard phantom with known densities. Tibial length (Le) was measured as the distance from the distal aspect of the medial malleolus to the proximal medial joint line. Axial scans of the nondominant leg $(2.2 \mathrm{~mm}$ thickness, $0.5 \mathrm{~mm}$ in-plane pixel size) were acquired at the $38 \%$ and $66 \%$ sites (measured relative to the distal endplate). Crosssectional morphology and tissue mineral density were quantified using Matlab software (MathWorks, Natick, MA USA). Images were rotated to standardize image orientation and thresholded to delineate bone $\left(800-1500 \mathrm{mg} / \mathrm{cm}^{3}\right)$ from nonbone voxels. Cortical tissue mineral density (TMD) was assessed for each crosssection by converting grayscale values to TMD using calibration constants. Morphological traits included the total cross-sectional area (Tt.Ar), cortical area (Ct.Ar), marrow area (Ma.Ar), area moments of inertia about the anteroposterior $\left(\mathrm{I}_{\mathrm{AP}}\right)$ and mediolateral axes $\left(\mathrm{I}_{\mathrm{ML}}\right)$, and polar moment of inertia $(J)$. Robustness was calculated as $\mathrm{Tt}$.Ar/Le to reflect the biological relationship between growth in width (which increases by area) and growth in length. All morphological analyses were conducted by the same individual $(\mathrm{CN})$ using BAMpack software (Bone Alignment and Measurement package; developed by L-3 ATI under contract with the U.S. Army Medical Research and Materiel Command). Some data were reported previously. ${ }^{(17-20)}$

\section{Functional adaptation}

Variation in bone mechanical functionality is traditionally assessed by plotting a morphological trait that is related to whole-bone stiffness (eg, section modulus) against a measure of body size (eg, body weight, lean mass, body mass index [BMI], or body weight times bone length). ${ }^{(21-24)}$ Impaired function could then be measured as a bone that is less stiff relative to body size. Herein, whole-bone stiffness was calculated indirectly from CT data as the product of tissue-modulus $(\mathrm{E})$ and the cross-sectional area moment of inertia (I), as described. ${ }^{(8)}$ The bending stiffness in the anteroposterior (A-P) direction was used (ie, bending about the mediolateral axis), because tibias are predominantly loaded in this direction during ambulation ${ }^{(25)}$ and show adaptive responses to exercise along this axis. ${ }^{(26)}$ Whole-bone stiffness (EI) was calculated from the pQCT images (38\% site) by converting TMD to $E$ and then multiplying $E$ by $I_{M L}$, which is the rectangular moment of inertia about the mediolateral axis. ${ }^{(8)}$ The product $\mathrm{El}$ was adjusted using the linear regression derived from a validation study that compared El estimated from PQCT with El measured directly from cadaveric tibiae subjected to conventional bending tests. ${ }^{(8)}$ The loads applied to a long 
bone can be estimated as a force (body weight) times a moment arm (tibial length). ${ }^{(21)}$ Bending stiffness, El, was plotted against body size (body weight $\times$ tibial length) and the residuals were calculated as a measure of the degree to which a bone was functionally adapted relative to body size (GraphPad Prism; La Jolla, CA, USA).

\section{Functional inequivalence}

To test whether the Israeli cohort showed functional inequivalence similar to that reported previously for the cohorts from the United Kingdom and the United States, ${ }^{(8)}$ we regressed bending stiffness (EI) against robustness after accounting for body size $(B W \times$ Le) effects by partial regression analysis (GraphPad Prism). The slope of the partial regression should not be significantly different from zero if slender and robust tibias exhibit the same stiffness relative to applied loads (ie, functional equivalence).

\section{Stress fracture risk}

A team of orthopedic surgeons examined the recruits every 2 to 3 weeks. Diagnosis of suspected stress fractures was done by radiography and scintigraphy. The odds ratio (OR) for individuals developing a stress fracture while having reduced tibial stiffness (EI) relative to body size (BW $\times$ Le) (ie, negative residual) was calculated (Minitab; State College, PA USA).

\section{Skeletal traits contributing to variation in functionality}

To test the hypothesis that reduced functionality arises in individuals that acquired fundamentally different sets of traits (robustness, Ct.Ar, TMD) by adulthood, we conducted a multivariate regression analysis followed by a K-means cluster analysis. We focused our attention on the relative proportions of robustness, Ct.Ar, and TMD, because we showed in prior work that these three traits are functionally related and contribute meaningfully to whole-bone stiffness. ${ }^{(8)}$ A multivariate regression analysis was conducted to determine the relative contributions of robustness, Ct.Ar, and TMD to the variation in tibial functionality as measured by the stiffness-body size residuals (Minitab). K-means cluster analysis was then used to segregate the male and female cohorts (separately) into subgroups based on the residuals for robustness when adjusted for body size, the residuals for Ct.Ar adjusted for body size and robustness, and the residuals for TMD adjusted for robustness (Minitab). Robustness was adjusted for body size ${ }^{(8)}$ to differentiate among individuals having slender or robust tibia for body size. Ct.Ar varies naturally with robustness (slender bones having less Ct.Ar compared to robust) and with body size. We adjusted for both factors to differentiate among individuals that have reduced (or greater) Ct.Ar for their robustness and body size. TMD was only adjusted for robustness, because TMD did not vary with body size. Using the residuals rather than absolute trait values in the cluster analysis was necessary to segregate individuals based on robustness and the degree to which Ct.Ar and TMD were adjusted relative to their robustness. This allowed us to relate mechanical functionality and fracture risk to specific underlying biological processes associated with functional adaptation. Residuals near zero indicate that the Ct.Ar or TMD was properly adjusted relative to robustness and/or body size, whereas negative or positive residuals indicate Ct.Ar or TMD was underadjusted or overadjusted, respectively. The analysis was conducted using standardized variables with the goal of identifying the maximum number of clusters that lead to unique sets of traits (ie, at least one trait from each cluster had to be different from the other clusters). We had no a priori knowledge of the number of structurally distinct subgroups that exist in the female and male cohorts. The number of clusters derived in this study was based on a statistical criteria such that the analyses were repeated while increasing the number of clusters until one or more clusters were assigned 3 or fewer individuals, compromising statistical power. Anthropometric and skeletal traits were averaged for each cluster and compared across clusters using ANOVA (GraphPad Prism). Comparisons between clusters was done using a Tukey post hoc test. The proportion of individuals with stress fractures in each cluster was determined.

\section{Results}

Differences between fracture and nonfracture groups

Confirmed stress fractures were observed in 15 women engaged in infantry training (7\%) and 23 men engaged in elite forces training (17\%). Anthropometric and morphological traits were compared between fracture and nonfracture cohorts (Table 1). Women with stress fractures were $7.5 \%$ heavier $(p<0.08)$, had $5.6 \%$ longer tibiae $(p<0.007)$, had a $13.3 \%$ greater $\mathrm{BW} \times$ Le $(p<0.02)$, and had a $12.4 \%$ greater tibial bending stiffness $(p<0.10)$ compared to the nonfracture cohort. However, women with stress fractures showed no differences in TMD, tissuemodulus, or morphology compared to the nonfracture group. In contrast, men with stress fractures were similar to men in the nonfracture group in terms of body size, but exhibited significantly different skeletal traits at the $38 \%$ site. The tibias for men with stress fractures were $5.3 \%$ more slender $(p<0.03)$, had an $11.4 \%$ smaller polar moment of inertia $(p<0.02)$, had a $0.8 \%$ greater TMD $(p<0.03)$, and an $11 \%$ lower whole-bone bending stiffness $(p<0.03)$ compared to men without stress fractures.

\section{Reduced stiffness contributes to stress fracture risk}

Whole-bone stiffness (EI) increased with body size $(B W \times$ Le) for both women and men, as expected (Fig. 1). Individuals with a stress fracture expressed the full range in body sizes, but had stiffness values that were near or below the regression line. For women undergoing light infantry training and men undergoing intense physical activity associated with elite combat training, having a negative residual from the stiffness-body size graph resulted in ORs of 1.5 (95\% confidence interval [Cl], 0.5-4.3) and $7.0(95 \% \mathrm{Cl}, 2.0-25.1)$, respectively, for developing a stress fracture. The sex-specific difference in ORs is consistent with the relative intensities of the training regimens.

\section{Functional inequivalence}

The partial regression between stiffness and robustness was significant for both women $\left(R^{2}=0.45, p<0.0001\right)$ and men $\left(R^{2}=0.27, p<0.0001\right)$ (not shown). The significant regressions confirmed that functional inequivalence exists for this cohort 
Table 1. Differences in Anthropometric and Skeletal Traits Between Fracture and Nonfracture Groups for Women Engaged in Light Infantry Training and for Men Engaged in Elite Forces Training

\begin{tabular}{|c|c|c|c|}
\hline Trait & Nonstress fracture & Stress fracture & $p$ \\
\hline \multicolumn{4}{|c|}{ Women engaged in light infantry training } \\
\hline$n$ & 189 & 15 & \\
\hline Body weight $(\mathrm{kg})$ & $58.9 \pm 9.3$ & $63.3 \pm 11.9$ & 0.08 \\
\hline Body height $(\mathrm{cm})$ & $162.0 \pm 6.4$ & $164.7 \pm 5.8$ & 0.15 \\
\hline BMI $\left(\mathrm{kg} / \mathrm{m}^{2}\right)$ & $22.6 \pm 3.3$ & $23.6 \pm 3.4$ & 0.30 \\
\hline Tibial length (mm) & $362.2 \pm 28.5$ & $381.0 \pm 23.6$ & 0.007 \\
\hline BW $\times$ Le $(\mathrm{kg} \mathrm{m})$ & $21.3 \pm 4.3$ & $24.2 \pm 5.3$ & 0.02 \\
\hline Stiffness, El $\left(\mathrm{Nmm}^{2}\right)$ & $167.2 \pm 45.1$ & $187.9 \pm 58.2$ & 0.10 \\
\hline \multicolumn{4}{|l|}{$38 \%$ site } \\
\hline Total area $\left(\mathrm{mm}^{2}\right)$ & $313.7 \pm 40.7$ & $326.0 \pm 43.6$ & 0.26 \\
\hline Cortical area $\left(\mathrm{mm}^{2}\right)$ & $234.0 \pm 32.4$ & $246.9 \pm 37.1$ & 0.16 \\
\hline Marrow area $\left(\mathrm{mm}^{2}\right)$ & $79.6 \pm 19.2$ & $79.1 \pm 16.9$ & 0.97 \\
\hline Polar moment of inertia $\left(\mathrm{mm}^{4}\right)$ & $17842 \pm 4745$ & $19784 \pm 5752$ & 0.13 \\
\hline $\mathrm{I}_{\mathrm{ML}}\left(\mathrm{mm}^{4}\right)$ & $11545 \pm 3159$ & $12954 \pm 3936$ & 0.10 \\
\hline $\mathrm{I}_{\mathrm{AP}}\left(\mathrm{mm}^{4}\right)$ & $6297 \pm 1784$ & $6830 \pm 2080$ & 0.27 \\
\hline Robustness (mm) & $0.87 \pm 0.10$ & $0.86 \pm 0.10$ & 0.60 \\
\hline TMD (mg HA) & $1186 \pm 18$ & $1183 \pm 19$ & 0.58 \\
\hline $\mathrm{E}(\mathrm{GPa})$ & $17.5 \pm 0.5$ & $17.4 \pm 0.6$ & 0.58 \\
\hline \multicolumn{4}{|l|}{$66 \%$ site } \\
\hline Total area $\left(\mathrm{mm}^{2}\right)$ & $447.1 \pm 71.3$ & $464.8 \pm 74.0$ & 0.37 \\
\hline Cortical area $\left(\mathrm{mm}^{2}\right)$ & $241.8 \pm 30.8$ & $244.8 \pm 45.0$ & 0.74 \\
\hline Marrow area $\left(\mathrm{mm}^{2}\right)$ & $205.3 \pm 60.7$ & $220.0 \pm 57.0$ & 0.38 \\
\hline Polar moment of inertia $\left(\mathrm{mm}^{4}\right)$ & $32936 \pm 8614$ & $35971 \pm 10898$ & 0.22 \\
\hline $\mathrm{I}_{\mathrm{ML}}\left(\mathrm{mm}^{4}\right)$ & $23235 \pm 6134$ & $25741 \pm 7623$ & 0.15 \\
\hline $\mathrm{I}_{\mathrm{AP}}\left(\mathrm{mm}^{4}\right)$ & $9698 \pm 2790$ & $10182 \pm 3598$ & 0.55 \\
\hline Robustness (mm) & $1.24 \pm 0.17$ & $1.23 \pm 0.18$ & 0.90 \\
\hline TMD (mg HA) & $1146 \pm 21$ & $1143 \pm 20$ & 0.58 \\
\hline $\mathrm{E}(\mathrm{GPa})$ & $16.3 \pm 0.6$ & $16.2 \pm 0.6$ & 0.58 \\
\hline \multicolumn{4}{|l|}{ Men engaged in elite forces training } \\
\hline$n$ & 109 & 23 & \\
\hline Body weight (kg) & $70.8 \pm 6.9$ & $70.2 \pm 6.0$ & 0.70 \\
\hline Body height $(\mathrm{cm})$ & $176.5 \pm 6.2$ & $177.0 \pm 5.7$ & 0.69 \\
\hline BMI $\left(\mathrm{kg} / \mathrm{m}^{2}\right)$ & $22.7 \pm 1.9$ & $22.4 \pm 1.4$ & 0.43 \\
\hline Tibial length (mm) & $422.6 \pm 26.0$ & $421.1 \pm 22.2$ & 0.80 \\
\hline BW $\times$ Le $(\mathrm{kg} \mathrm{m})$ & $30.0 \pm 3.9$ & $29.6 \pm 3.5$ & 0.69 \\
\hline Stiffness, El $\left(\mathrm{Nmm}^{2}\right)$ & $337.9 \pm 76.2$ & $300.8 \pm 48.1$ & 0.03 \\
\hline \multicolumn{4}{|l|}{$38 \%$ site } \\
\hline Total area $\left(\mathrm{mm}^{2}\right)$ & $444.6 \pm 48.7$ & $419.6 \pm 35.6$ & 0.02 \\
\hline Cortical area $\left(\mathrm{mm}^{2}\right)$ & $341.9 \pm 40.4$ & $331.3 \pm 25.4$ & 0.23 \\
\hline Marrow area $\left(\mathrm{mm}^{2}\right)$ & $102.7 \pm 28.5$ & $88.3 \pm 20.3$ & 0.02 \\
\hline Polar moment of inertia $\left(\mathrm{mm}^{4}\right)$ & $36289 \pm 7796$ & $32163 \pm 5151$ & 0.02 \\
\hline $\mathrm{I}_{\mathrm{ML}}\left(\mathrm{mm}^{4}\right)$ & $24133 \pm 5556$ & $21131 \pm 3388$ & 0.01 \\
\hline $\mathrm{I}_{\mathrm{AP}}\left(\mathrm{mm}^{4}\right)$ & $12090 \pm 985$ & $10720 \pm 642$ & 0.01 \\
\hline Robustness (mm) & $1.05 \pm 0.12$ & $1.00 \pm 0.09$ & 0.03 \\
\hline TMD (mg HA) & $1153 \pm 20$ & $1163 \pm 16$ & 0.03 \\
\hline $\mathrm{E}(\mathrm{GPa})$ & $16.5 \pm 0.6$ & $16.8 \pm 0.5$ & 0.03 \\
\hline \multicolumn{4}{|l|}{$66 \%$ site } \\
\hline Total area $\left(\mathrm{mm}^{2}\right)$ & $697.2 \pm 105.9$ & $667.2 \pm 100.8$ & 0.22 \\
\hline Cortical area $\left(\mathrm{mm}^{2}\right)$ & $324.2 \pm 43.1$ & $317.1 \pm 32.3$ & 0.46 \\
\hline Marrow area $\left(\mathrm{mm}^{2}\right)$ & $373.0 \pm 105.7$ & $350.1 \pm 100.8$ & 0.34 \\
\hline Polar moment of inertia $\left(\mathrm{mm}^{4}\right)$ & $71622 \pm 15951$ & $65495 \pm 12756$ & 0.09 \\
\hline $\mathrm{I}_{\mathrm{ML}}\left(\mathrm{mm}^{4}\right)$ & $50925 \pm 11796$ & $46011 \pm 8900$ & $\begin{array}{r}0.06 \\
\text { inued) }\end{array}$ \\
\hline
\end{tabular}


Table 1. (Continued)

\begin{tabular}{lccc}
\hline Trait & Nonstress fracture & Stress fracture & $p$ \\
\hline $\mathrm{I}_{\mathrm{AP}}\left(\mathrm{mm}^{4}\right)$ & $20697 \pm 5057$ & $19484 \pm 4760$ & 0.29 \\
Robustness $(\mathrm{mm})$ & $1.65 \pm 0.23$ & $1.58 \pm 0.20$ & 0.19 \\
$\mathrm{TMD}(\mathrm{mg} \mathrm{HA})$ & $1101 \pm 22.2$ & $1109 \pm 19.3$ & 0.13 \\
$\mathrm{E}(\mathrm{GPa})$ & $14.9 \pm 0.7$ & $15.2 \pm 0.6$ & 0.13 \\
\hline
\end{tabular}

Data are presented as mean \pm SD.

$\mathrm{BMI}=$ body mass index; $\mathrm{BW}=$ body weight; $\mathrm{Le}=$ bone length; $\mathrm{El}=$ bending stiffness; $\mathrm{I}_{\mathrm{ML}}=$ moment of inertia about the mediolateral axes; $\mathrm{I}_{\mathrm{AP}}=$ moment of inertia about the anteroposterior; $\mathrm{TMD}=$ tissue mineral density; $\mathrm{HA}=$ hydroxyapatite; $\mathrm{E}=$ tissue-modulus.

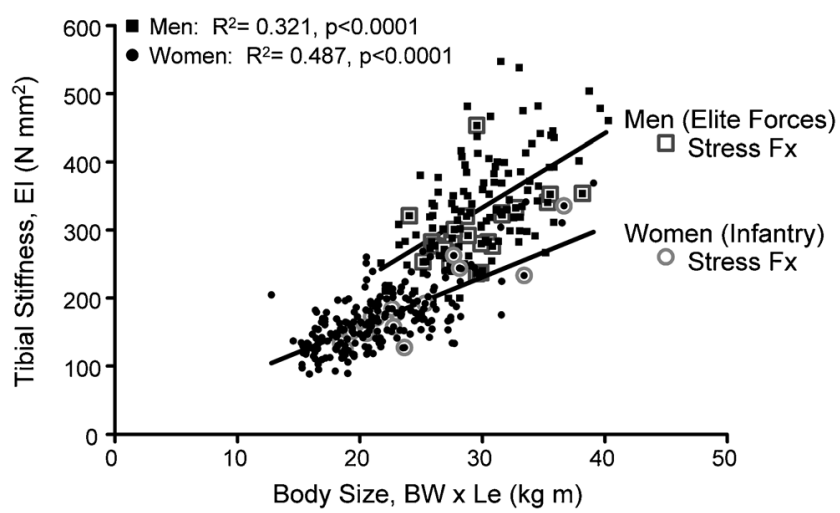

Fig. 1. Tibial stiffness increased with body size $(B W \times$ Le), as expected. Men showed greater stiffness compared to women for any given body size (ANCOVA: slope $p<0.006$ ). Individuals with confirmed stress fractures fell near or below the linear regression, suggesting that having reduced stiffness relative to body size may contribute to stress fracture risk.

and indicated that slender tibias were as much as two times less stiff for body size compared to robust tibias. The slope ( $p=0.83$ ) and $y$-intercept $(p=0.82$ ) of the partial regressions did not differ between sexes (analysis of covariance [ANCOVA]).

Functionality and fracture risk can arise from the acquisition of different sets of traits

To test the hypothesis that reduced functionality and fracture risk arise in individuals that acquired different sets of traits (i.e., robustness, Ct.Ar, TMD) by adulthood, we first assessed the relative contributions of robustness, Ct.Ar, and TMD to the variation in tibial functionality (ie, stiffness relative to body size). A multiple regression analysis revealed that $69 \%$ to $72 \%$ of the variation in the stiffness-body size residuals was explained by the residuals for robustness, Ct.Ar, and TMD (Table 2 ). The robustness and Ct.Ar residuals were significant determinants of the stiffness-body size residuals for women and men, indicating that the natural variation in robustness and the variation in the extent to which the system adjusted Ct.Ar were the primary determinants of the interindividual variation in mechanical function.

Individuals were then segregated into clusters based on the residuals for robustness, $\mathrm{Ct} . \mathrm{Ar}$, and TMD, and then each of these traits was compared across clusters using ANOVA. The women segregated into eight clusters and the men into seven clusters before one or more clusters were assigned 3 or fewer members. The clusters were arranged relative to the average stiffness-body size residual, such that Cluster 1 was assigned individuals with the lowest stiffness relative to body size and Cluster 8 (women) and Cluster 7 (men) were assigned individuals with the highest stiffness relative to body size. There were no differences in body size (BW $\times$ Le), body weight (not shown), or height (not shown) among clusters (Fig. 2). The number of individuals assigned to each cluster is shown in Fig. 2A,B. Tibial stiffness and the stiffness-body size residuals were shown to convey the magnitude of stiffness variation that exists among the clusters. There was a $42 \%$ difference in the absolute value of stiffness between Clusters 1 and 8 for women and a 37\% difference between Clusters 1 and 7 for men.

Figure 3 (women) and Fig. 4 (men) show the absolute values for robustness, Ct.Ar, and TMD, as well as the residuals for each trait. All traits showed significant differences across clusters ( $p<0.0001$, ANOVA). Individuals were assigned to clusters with unique combinations of traits (robustness, Ct.Ar, TMD) and this was illustrated schematically in Fig. 5. The variation in functionality largely reflected the variation in robustness, with a few exceptions that could be attributed to variation in the degree to which Ct.Ar and/or TMD were adjusted.

The concept that functionality can arise from the acquisition of different sets of traits is illustrated by comparing Clusters 1 and 2 for individuals with low functionality and Clusters 7 and 8

Table 2. Multiple Linear Regression Analysis Between El-BWLe Res and Rob Res, Ct.Ar Res, and TMD Res

\begin{tabular}{|c|c|c|c|}
\hline Sex & Equation & $R^{2}$-adj & $p$ \\
\hline M & El-BWLe Res $=0.70+\mathbf{4 1 1}$ Rob Res $+\mathbf{0 . 9 5} \mathbf{C t A r}$ Res -0.13 TMD Res & 72.1 & 0.0001 \\
\hline $\mathrm{F}$ & El-BWLe Res $=0.31+\mathbf{2 4 7}$ Rob Res $+\mathbf{0 . 7 8} \mathbf{C t A r}$ Res $+0.01 \mathrm{TMD}$ Res & 68.9 & 0.0001 \\
\hline
\end{tabular}

All traits were measured at the $38 \%$ anatomical site. Bold indicates components contributing significantly to the regression.

El-BWLe Res = residuals calculated from the stiffness-body size regression; Rob Res = residuals calculated for bone robustness; Ct.Ar Res $=$ cortical area residual; TMD Res $=$ tissue-mineral density residual. 
A

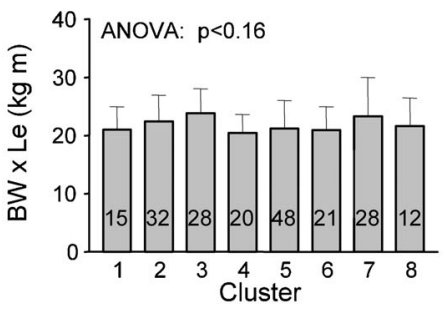

B

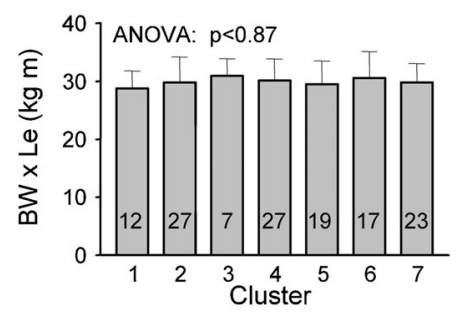

C

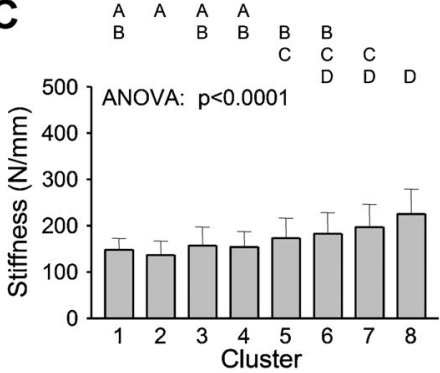

D

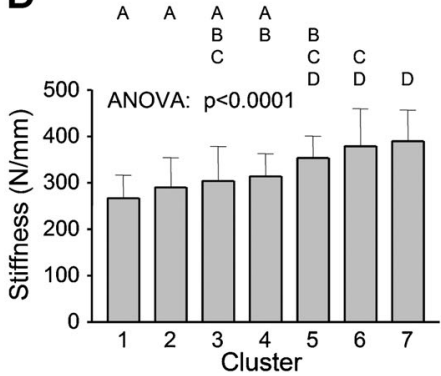

E

$\begin{array}{lllll}A & A & A & & \\ & B & B & B & \\ & & & C & C\end{array}$

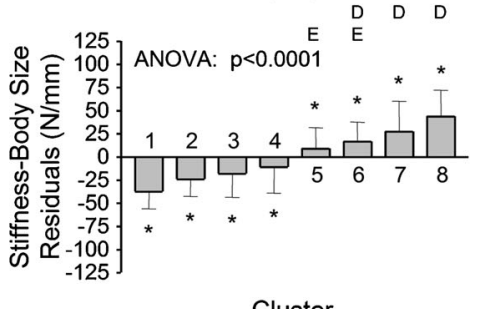

Cluster

$\mathbf{F}$

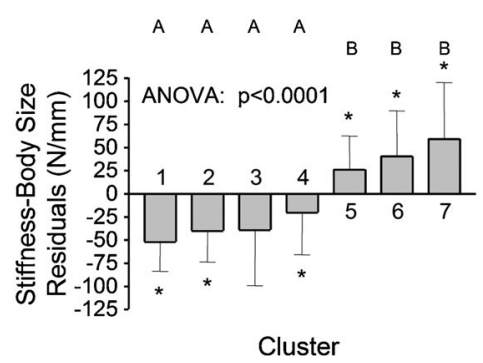

Fig. 2. Women and men were segregated into clusters based on the residuals for tibial robustness, cortical area, and TMD. Body size, tibial stiffness, and the measure of mechanical functionality (stiffness-body size residuals) were compared across clusters for women $(A, C, E)$ and men $(B, D, F)$. The clusters were arranged from lowest to highest functionality. The * in $E$ and $F$ indicate clusters with residuals that were significantly different from zero $(p<0.05$, Wilcoxon signed rank test). No difference in body size was found among the clusters. An ANOVA was conducted for each trait to test for differences among clusters. Clusters with letters in common indicate no differences ( $p>0.05$, Tukey post hoc test).

(women) or Clusters 6 and 7 (men) for individuals with high functionality. For women, reduced functionality could arise for tibia with an average robustness combined with a lower than expected Ct.Ar and TMD for this robustness (Cluster 1) or slender tibia combined with the expected value for Ct.Ar and a higher than expected value for TMD (Cluster 2). Higher functionality could arise for robust tibia combined with the expected amount of Ct.Ar and higher than expected TMD for this robustness
A

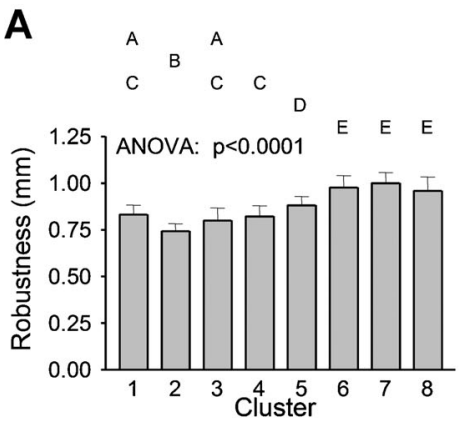

B

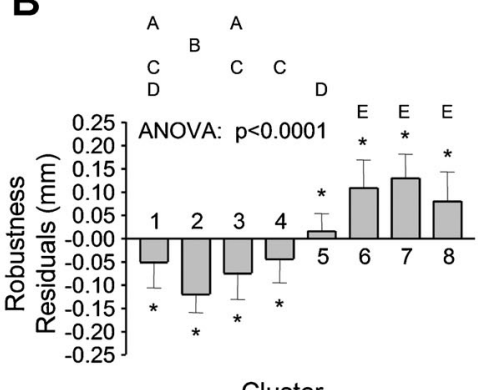

C

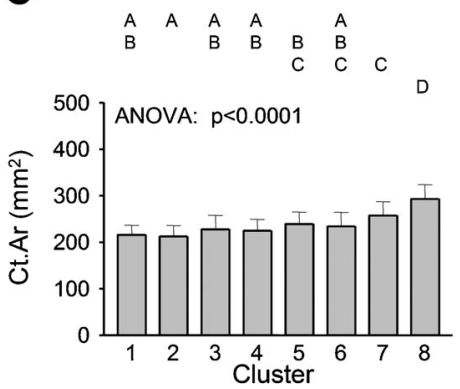

D

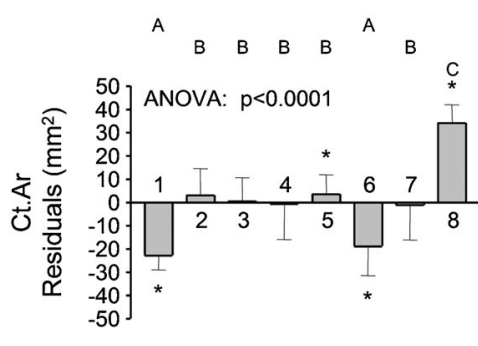

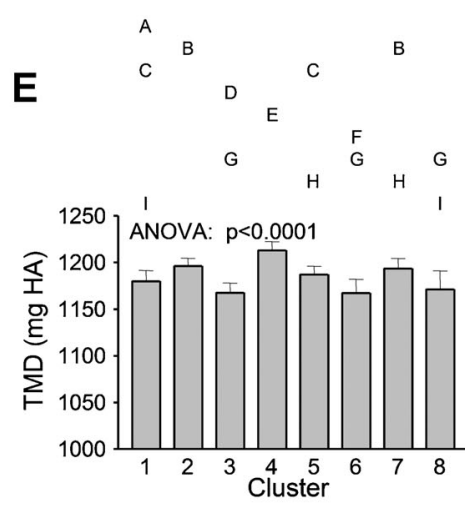

$\mathbf{F}$

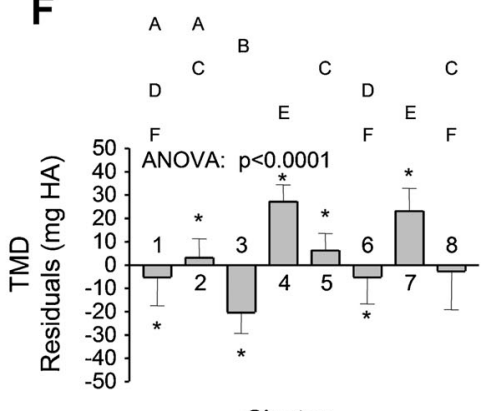

Cluster

Cluster

Cluster

Fig. 3. Tibias for young adult women engaged in infantry training were segregated into eight clusters and the average $(A)$ robustness, $(C)$ cortical area, and $(E)$ TMD for each cluster was calculated; $(B, D, F)$ the residuals for each of these traits. The * indicates clusters with residuals that were significantly different from zero ( $p<0.05$, Wilcoxon signed rank test). An ANOVA was conducted for each trait to test for differences among clusters. Clusters with letters in common indicate no differences ( $p>0.05$, Tukey post hoc test). 
A

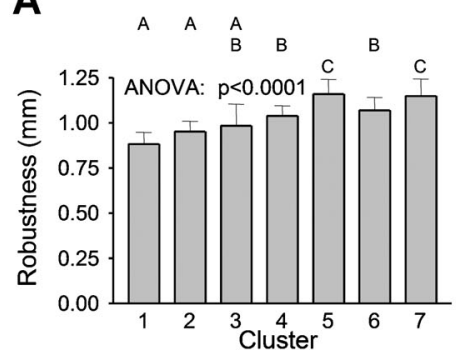

B

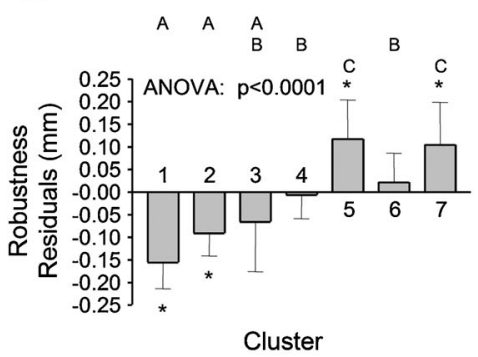

C
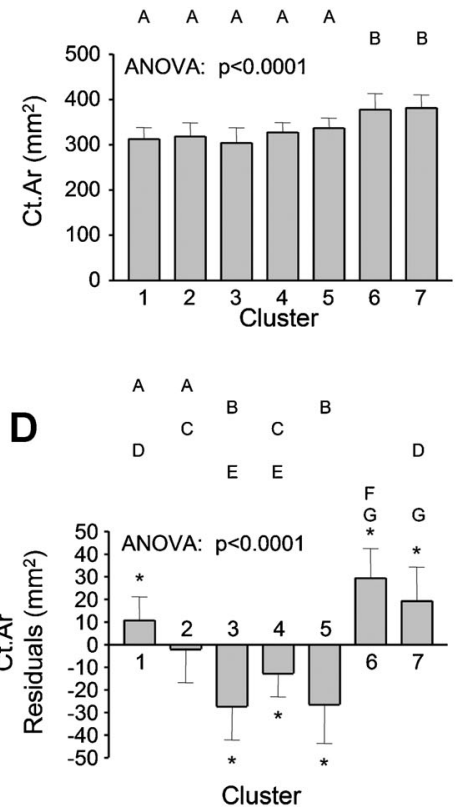

B
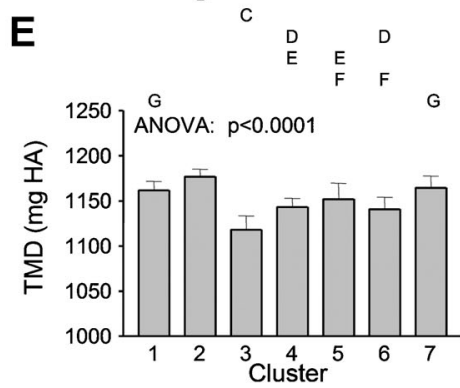

$\mathbf{F}$

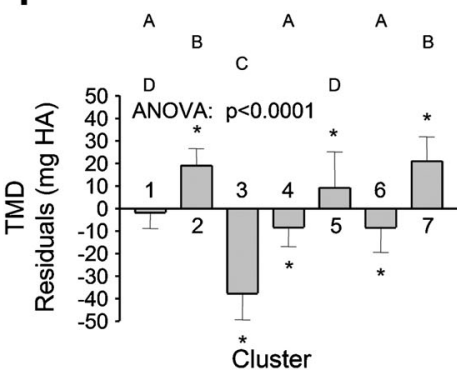

Fig. 4. Tibias for young adult men engaged in elite forces training were segregated into seven clusters and the average $(A)$ robustness, $(C)$ cortical area, and $(E)$ TMD for each cluster was calculated; $(B, D, F)$ the residuals for each of these traits. The * indicates clusters with residuals that were significantly different from zero ( $p<0.05$, Wilcoxon signed rank test). An ANOVA was conducted for each trait to test for differences among clusters. Clusters with letters in common indicate no differences ( $p>0.05$, Tukey post hoc test).

(Cluster 7) or robust tibia combined with higher than expected Ct.Ar but the expected TMD (Cluster 8). For men, reduced functionality could arise for slender tibia combined with a higher than expected Ct.Ar and the expected TMD (Cluster 1) or slender tibia combined with the expected Ct.Ar and higher than expected TMD (Cluster 2). Higher functionality could arise for tibia with an average robustness combined with higher than expected Ct.Ar and lower than expected values for TMD
(Cluster 6) or robust tibia combined with higher than expected values for both Ct.Ar and TMD (Cluster 7).

Stress fractures can arise from the acquisition of different sets of traits

The proportion of men in each cluster with stress fractures is shown in Fig. 6. This analysis was conducted for men only, since women did not show a sufficiently large number of stress

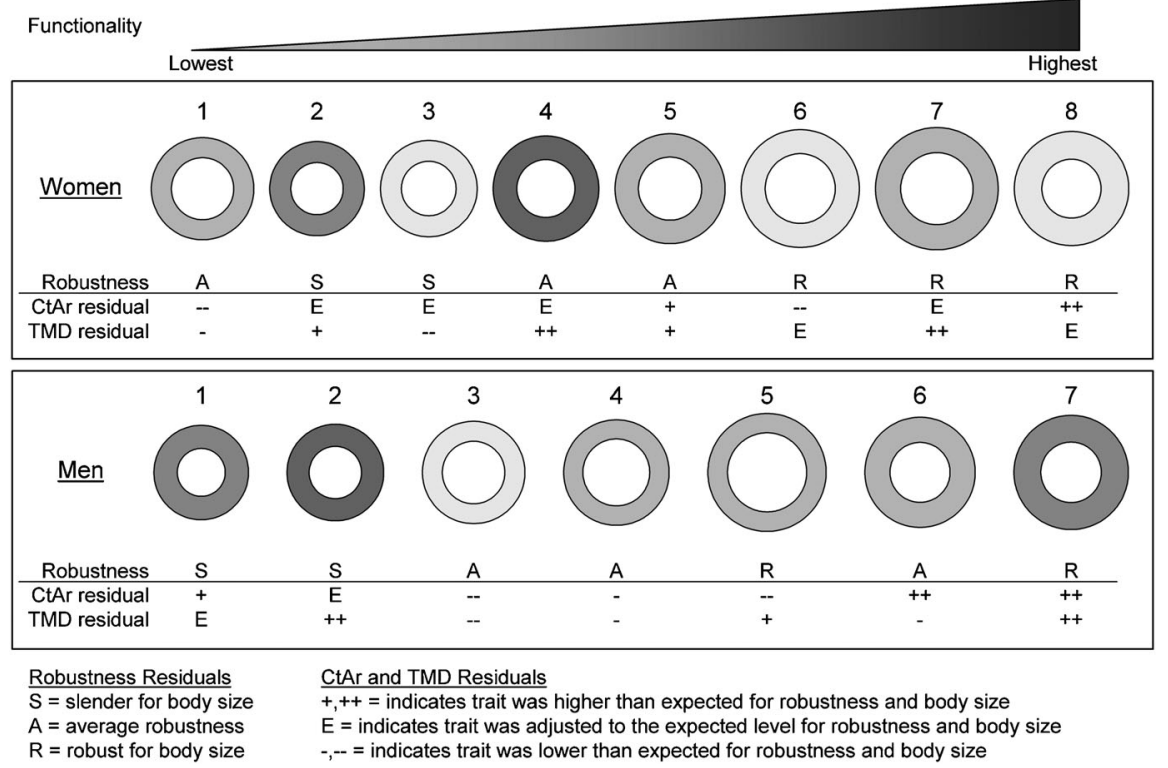

Fig. 5. Schematic depicting the average trait sets for each of the clusters. The cross-sectional images are shown as cylindrical to simplify the appearance. The outer and inner surfaces and the grayscale, which is proportional to TMD, were based on the average traits shown in Figs. 3 and 4. 


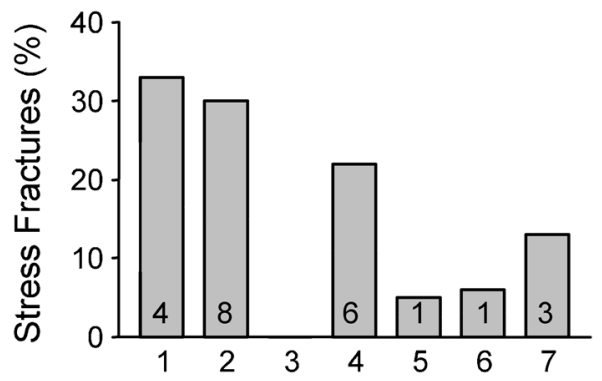

Fig. 6. The percentage of men in each cluster that developed a stress fracture during military training. The number of stress fracture cases in each cluster is also indicated.

fractures to draw a meaningful conclusion from this analysis. For men engaged in elite combat training, the distribution of stress fractures among the seven clusters was borderline significantly different from the null hypothesis that the number of fractures cases would be uniformly distributed among the clusters $(p<0.09$, chi-square test). However, stress fracture cases segregated dominantly to Clusters 1, 2, and 4, together accounting for $78 \%$ of the total number of cases $(p<0.03$, chisquare test).

\section{Discussion}

Tibial stiffness estimated from pQCT images and confirmed stress fracture incidence obtained for young adult women and men engaged in military training were used to test the hypotheses that reduced tibial functionality (ie, stiffness relative to body size) can arise from the acquisition of biologically distinct sets of traits and that individuals with reduced tibial functionality will show an increased risk of fracturing. The biologically distinct sets of traits included robustness adjusted for body size and Ct.Ar and TMD adjusted for robustness adjusted for body size, and Ct.Ar and TMD adjusted for robustness and body size. Mechanical functionality was assessed in the traditional manner by relating whole-bone stiffness to body size ${ }^{(21,27,28)}$ and quantified on an individualized basis by calculating the residuals from this linear regression as a measure of how stiff each person's bone was relative to their body size. Variation in this stiffness-body size residual should be proportional to the variation in peak strains engendered during daily loading conditions. The variation in tibial functionality reported in Fig. 2 is representative of healthy, young adult women and men. Including individuals from the general population would be expected to increase the amount of functional variation across the range in tibial robustness.

Although functionality was assessed in a traditional manner, we used a novel approach to identify the bone traits contributing to the interindividual variation in functionality and fracture risk. Our systems-based approach provided a new way to think about functional adaptation, because it allowed us to relate the variation in whole-bone stiffness to biologically distinct aspects of the functional adaptation process. Bone mineral density (BMD), moment of inertia, and section modulus are mechanically relevant traits but provide little insight into the biological mechanisms contributing to the variation in bone functionality. In addition to examining absolute trait values and body size- adjusted traits, we also assessed tibial robustness and the degree to which Ct.Ar and TMD were adjusted relative to the natural variation in robustness. This was necessary because prior work showed that variation in whole-bone stiffness arises from variation in Ct.Ar and TMD superimposed on the variation in robustness. ${ }^{(8)}$ To account for this "tiered" variation, we adjusted Ct.Ar and TMD to evaluate whether either of these traits was underadjusted (residual $<0$ and significantly different from zero), properly adjusted (residual not different from zero), or overadjusted (residual $>0$ and significantly different from zero) for each person's robustness and body size. Robustness is a measure of the amount of periosteal expansion relative to growth in length. Ct.Ar is a measure of the amount of bone accrued during growth. TMD is a measure of matrix mineralization and the porosity associated with intracortical remodeling. ${ }^{(8)}$ Robustness, Ct.Ar, and TMD together accounted for $69 \%$ to $72 \%$ of the variation in tibial functionality (Table 2). A K-means cluster analysis successfully segregated individuals into subgroups based on the residuals for robustness, Ct.Ar, and TMD. An ANOVA confirmed that the clusters exhibited significantly different functionalities (ie, stiffness relative to body size) as well as unique combinations of functionally related traits (Fig. 5). This outcome supported the hypothesis that the variation in bone function among healthy individuals arises from the acquisition of fundamentally different sets of traits. This means that two people can show similar reductions in strength, but that they got to this strength value in different ways. Individual trait sets may provide clues to different biological pathways leading to reduced strength.

Importantly, a comparison of adjacent clusters indicated that the clusters were distinguished not by small shifts in relative trait values, but in some cases by sets of traits arising from alternative ways in which Ct.Ar and TMD could be adjusted relative to robustness. It is reasonable to speculate that clusters with residuals not different from zero would be considered welladapted, whereas clusters with negative residuals for Ct.Ar and TMD which are significantly different from zero may indicate impaired functional adaptation. Clusters with reduced residuals for Ct.Ar may indicate impaired accrual of bone during growth. Clusters with reduced TMD residuals may indicate reduced mineralization and/or increased intracortical porosity. Further, the combination of robustness, Ct.Ar, and TMD may reveal compensatory interactions among these traits. For example, the trait set exhibited by men in Cluster 5 suggested that a lower than expected cortical area may be compensated by a higher than expected TMD, or vice versa. Although the causal nature of these trait interactions is unknown, our analysis does indicate that interactions among these three traits provides tremendous flexibility for establishing whole-bone mechanical function. This outcome provides important insight into alternative biological mechanisms that can be used to establish mechanical homeostasis within the skeletal system and argues against the use of a single trait (eg, bone width, Ct.Ar, cortical thickness, moment of inertia) to presume bone functionality, which is often done.

Flexibility in establishing mechanical functionality allows complex adaptive systems to tolerate many genetic and/or environmental variants. ${ }^{(29-34)}$ Environmental and genetic factors 
impairing skeletal growth and leading to reduced Ct.Ar and/or TMD are arguably an expected source of reduced functionality. However, our analysis identified multiple sources of reduced functionality. We confirmed that the cohort of Israeli women and men also exhibited natural variation in stiffness relative to robustness (ie, functional inequivalence), consistent with prior work. $^{(8)}$ All individuals in our study population were healthy young adults that passed rigorous medical exams. Thus, the twofold difference in stiffness between slender and robust tibias was not pathological but simply a natural variant arising from intrinsic limitations in the skeletal systems' ability to fully compensate the nonlinear relationship between bone width and whole-bone stiffness. ${ }^{(8)}$ Because the trait sets in each cluster arise through different biological pathways (eg, slenderness versus reduced (t.Ar versus reduced TMD), our analysis indicated there are multiple biological mechanisms contributing to the variation in bone strength. Distinguishing among these biological mechanisms may benefit efforts aimed at identifying genetic or environmental factors that impair or promote the ability of the skeletal system to grow and maintain a mechanically functional skeletal structure.

What is important and novel in this study is that the variation in functionality occurred either with or without impaired functional adaptation. Tibias in Cluster 2 for women and Clusters 1 and 2 for men exhibited reduced functionalities, but we would argue that these tibias were well-adapted structures given that they had the expected (or a greater) amount of Ct.Ar and TMD for their robustness and body size. These clusters exhibited some of the highest TMD values and the lowest marrow areas (not shown) and it is unclear whether tissue-level mechanical properties or Ct.Ar could be adjusted any further. Thus, individuals in these particular clusters appeared to achieve the highest stiffness possible for their robustness. Thus, a person can be considered to have well-adapted tibias and yet still show reduced functionality. This is a major outcome of this study. The functional inequivalence associated with the natural variation in robustness results in slender bones being as much as two times less stiff for body size as robust bones. Efforts to reduce stress fracture incidence in these individuals may be better served by modifying training regimens to reduce tissue-level strains and damage accumulation (eg, reducing exercise intensity and weekly mileage) rather than trying to strengthen a well-adapted bone using adaptation-directed interventions. ${ }^{(20)}$

Although flexibility in assembling traits to accommodate genetic and environmental perturbations is critical for the functioning of complex adaptive systems, the downside is that certain trait sets may perform better than others under extreme loading conditions. Our analysis supported the hypothesis that having reduced stiffness relative to body size was associated with an increased risk of developing a stress fracture during military training. It would be difficult to attribute the differences in $O R$ between women $(O R=1.5)$ and men $(O R=7)$ for developing a stress fracture to a sex-effect given the relatively small number of stress fracture cases observed for women and the differences in physical activity during basic training. Women generally sustain approximately five times more stress fractures than men during gender-integrated military training regimens. ${ }^{(35)}$ However, women in our study were engaged in light infantry training, which is considered rigorous but may not subject the skeleton to the extreme loading conditions expected for men engaged in elite-forces combat training.

Finding that reduced stiffness is a major determinant of stress fractures was not surprising. ${ }^{(1,36,37)}$ The important contribution here was finding that reduced stiffness arises through biologically distinct pathways. For men engaged in elite-forces training, $78 \%$ of the fracture cases ( $p<0.03$, chi-square) segregated into clusters exhibiting increased bone slenderness (Clusters 1 and 2) and impaired functional adaptation (Cluster 4). Slender tibias with reduced stiffness relative to body size would be expected to experience greater tissue strains and to accumulate a greater amount of damage during basic training. The higher absolute magnitude of TMD for Clusters 1 and 2 could result from increased mineralization and/or reduced intracortical porosity arising from suppressed internal remodeling, ${ }^{(8)}$ both of which may exacerbate damage accumulation during intensive training. Stress fractures were not limited to individuals with slender bones, but a high proportion (22\%) of individuals in Cluster 4 had stress fractures with a modest decrease in functionality. We interpret the trait set of Cluster 4 to indicate a failure to accumulate adequate Ct.Ar during growth and that the matrix is either less mineralized or more porous (or both). Lack of fracture cases in Cluster 3 was likely a result of the low number of individuals $(n=7)$ segregating into this cluster. Larger databases are needed to draw firm conclusions regarding the types of trait sets that show increased stress fracture risk.

A limitation of the K-means cluster analysis is that we discretized continuous data. This does not mean that biological processes underlying functional adaptation act in a discretized manner. This discretization was done primarily to advance the idea that biomechanically and biologically distinct subgroups exist within a population. Although our sample size was sufficiently large to establish eight independent clusters for women and seven for men, the actual number of clusters achievable is unknown. Our criterion for determining the number of independent clusters was based on maintaining a modest degree of statistical power to test for independent trait sets and does not reflect the actual number of clusters that may be identified using a larger database. Expanding this work to a more general population will likely identify additional types of trait sets, particularly those with reduced functionality.

The two major outcomes of this study are that tibias with reduced stiffness for body size are at increased risk of developing stress fractures during military training and that variation in tibial functionality among healthy young adult women and men arises from the acquisition of biologically distinct sets of traits (robustness, Ct.Ar, TMD). How these concepts translate to the aging skeleton will be important to determine. Most studies aim to identify a single trait or a small group of traits that increase fracture risk. This traditional approach provides a simple diagnostic that can be used clinically to identify individuals at increased risk of fracturing. However, our work shows that a single trait is not sufficient to predict functionality and that diagnostics should take the complex adaptive nature of the skeletal system into consideration. Given that similar levels of functionality can arise from different biological sources, indiscriminant assessment of genomic or gene expression data 
based on absolute trait values could be problematic when attempting to identify factors that are responsible for reduced functionality and increased fracture risk. Knowing that multiple sources contribute to the variation in mechanical functional has important implications for advancing our ability to develop personalized preventative diagnostics and treatments aimed at strengthening bone.

\section{Disclosures}

All authors state that they have no conflicts of interest.

\section{Acknowledgements}

This work was supported by grants from the U.S. Department of Defense (W81XWH-09-2-0113, W81XWH-07-C-0097) and an medical research and material command (MRMC) Bone Health Grant. The opinions or assertions contained herein are the private views of the authors and are not to be construed as official or as reflecting the views of the U.S. Army or the Department of Defense.

Authors' roles: All authors contributed in a significant way to the generation of this manuscript. This effort includes hypothesis generation (KJJ, DSM, RE, and CHN), data collection and analysis (DSM, TE, AH, RY, RE, CHN, and $A C_{\text {, }}$ ), statistical analysis (KJJ and $J J G$ ), and manuscript preparation (KJJ, DSM, RE, and CHN).

\section{References}

1. Szulc P, Munoz F, Duboeuf F, Marchand F, Delmas PD. Low width of tubular bones is associated with increased risk of fragility fracture in elderly men-the MINOS study. Bone. 2006;38(4):595-602.

2. Duan Y, Beck TJ, Wang XF, Seeman E. Structural and biomechanical basis of sexual dimorphism in femoral neck fragility has its origins in growth and aging. J Bone Miner Res. 2003;18(10):1766-74.

3. Tommasini SM, Nasser P, Schaffler MB, Jepsen KJ. Relationship between bone morphology and bone quality in male tibias: implications for stress fracture risk. J Bone Miner Res. 2005;20 (8):1372-80.

4. Tommasini SM, Nasser P, Hu B, Jepsen KJ. Biological co-adaptation of morphological and composition traits contributes to mechanical functionality and skeletal fragility. J Bone Miner Res. 2008;23(2):23646.

5. Tommasini SM, Nasser $P$, Jepsen KJ. Sexual dimorphism affects tibia size and shape but not tissue-level mechanical properties. Bone. 2007;40(2):498-505.

6. Pandey N, Bhola S, Goldstone A, Chen F, Chrzanowski J, Terranova CJ, Ghillani R, Jepsen KJ. Inter-individual variation in functionally adapted trait sets is established during post-natal growth and predictable based on bone robusticity. J Bone Miner Res. 2009;24 (12):1969-80.

7. Bhola S, Chen J, Fusco J, Duarte GF, Andarawis-Puri N, Ghillani R, Jepsen KJ. Variation in childhood skeletal robustness is an important determinant of cortical area in young adults. Bone. 2011;49(4):799809.

8. Jepsen KJ, Centi A, Duarte GF, Galloway K, Goldman H, Hampson N, Lappe JM, Cullen DM, Greeves J, Izard R, Nind IC, Kraemer WJ, Negus $\mathrm{CH}$, Evans RK. Biological constraints that limit compensation of a common skeletal trait variant lead to inequivalence of tibial function among healthy young adults. J Bone Miner Res. 2011;26(12):2872-5.

9. Albright F, Smith PH, Richardson AM. Post-menopausal osteoporosis. Its clinical features. JAMA. 1941;116:2465-74.
10. Landin L, Nilsson BE. Bone mineral content in children with fractures. Clin Orthop Relat Res. 1983;178:292-6.

11. Milgrom C, Giladi M, Simkin A, Rand N, Kedem R, Kashtan H, Stein M, Gomori M. The area moment of inertia of the tibia: a risk factor for stress fractures. J Biomech. 1989;22(11-12):1243-8.

12. Franklyn M, Oakes B, Field B, Wells P, Morgan D. Section modulus is the optimum geometric predictor for stress fractures and medial tibial stress syndrome in both male and female athletes. Am J Sports Med. 2008;36(6):1179-89.

13. Jepsen KJ, Courtland H-W, Nadeau JH. Genetically-determined phenotype covariation networks control bone strength. J Bone Miner Res. 2010;25(7):1581-93.

14. Rivadeneira F, Zillikens MC, De Laet CE, Hofman A, Uitterlinden AG, Beck TJ, Pols HA. Femoral neck BMD is a strong predictor of hip fracture susceptibility in elderly men and women because it detects cortical bone instability: the Rotterdam Study. J Bone Miner Res. 2007;22(11):1781-90.

15. LaCroix AZ, Beck TJ, Cauley JA, Lewis CE, Bassford T, Jackson R, Wu G, Chen Z. Hip structural geometry and incidence of hip fracture in postmenopausal women: what does it add to conventional bone mineral density?. Osteoporos Int. 2010;21(6):919-29.

16. Biewener AA. Safety factors in bone strength. Calcif Tissue Int. 1993; 53(Suppl 1):S68-74.

17. Evans RK, Negus C, Antczak AJ, Yanovich R, Israeli E, Moran DS. Sex differences in parameters of bone strength in new recruits: beyond bone density. Med Sci Sports Exerc. 2008; 40(11 Suppl):S645-53.

18. Moran DS, Israeli E, Evans RK, Yanovich R, Constantini N, Shabshin N, Merkel D, Luria O, Erlich T, Laor A, Finestone A. Prediction model for stress fracture in young female recruits during basic training. Med Sci Sports Exerc. 2008; 40(11 Suppl):S636-44.

19. Finestone A, Milgrom C, Evans R, Yanovich R, Constantini N, Moran DS. Overuse injuries in female infantry recruits during low-intensity basic training. Med Sci Sports Exerc. 2008; 40(11 Suppl):S630-5.

20. Moran DS, Finestone A, Arbel Y, Shabshin N, Laor A. Simplified model to predict stress fracture in young elite combat recruits. J Strength Cond Res. 2011;26(9):2585-92.

21. Selker F, Carter DR. Scaling of long bone fracture strength with animal mass. J Biomech. 1989;22(11-12):1175-83.

22. van der Meulen MC, Ashford MW Jr, Kiratli BJ, Bachrach LK, Carter DR. Determinants of femoral geometry and structure during adolescent growth. J Orthop Res. 1996;14(1):22-9.

23. Ruff C. Growth in bone strength, body size, and muscle size in a juvenile longitudinal sample. Bone. 2003;33(3):317-29.

24. Petit MA, Beck TJ, Shults J, Zemel BS, Foster BJ, Leonard MB. Proximal femur bone geometry is appropriately adapted to lean mass in overweight children and adolescents. Bone. 2005;36(3):568-76.

25. Peterman MM, Hamel AJ, Cavanagh PR, Piazza SJ, Sharkey NA. In vitro modeling of human tibial strains during exercise in micro-gravity. J Biomech. 2001;34(5):693-8.

26. Macdonald HM, Cooper DM, McKay HA. Anterior-posterior bending strength at the tibial shaft increases with physical activity in boys: evidence for non-uniform geometric adaptation. Osteoporos Int. 2009;20(1):61-70.

27. Sumner DR, Andriacchi TP. Adaptation to differential loading: comparison of growth-related changes in cross-sectional properties of the human femur and humerus. Bone. 1996;19(2):121-6.

28. Ruff C. Growth tracking of femoral and humeral strength from infancy through late adolescence. Acta Paediatr. 2005;94(8):1030-7.

29. Waddington $\mathrm{CH}$. Canalization of development and the inheritance of acquired characters. Nature. 1942;14:563-5.

30. Olson EC, Miller RL. Morphological Integration. Chicago: The University of Chicago Press Ltd; 1958.

31. Cheverud JM. Developmental integration and the evolution of pleiotropy. Am Zool. 1996;36:44-50. 
32. Nadeau JH, Burrage LC, Restivo J, Pao YH, Churchill G, Hoit BD. Pleiotropy, homeostasis, and functional networks based on assays of cardiovascular traits in genetically randomized populations. Genome Res. 2003;13(9):2082-91.

33. Marder E, Goaillard JM. Variability, compensation and homeostasis in neuron and network function. Nat Rev Neurosci. 2006;7(7): 563-74.

34. Nowlan NC, Prendergast PJ. Evolution of mechanoregulation of bone growth will lead to non-optimal bone phenotypes. J Theor Biol. 2005;235(3):408-18.
35. Friedl KE. Biomedical research on health and performance of military women: accomplishments of the Defense Women's Health Research Program (DWHRP). J Womens Health (Larchmt). 2005;14(9):764-802.

36. Beck TJ, Ruff CB, Mourtada FA, Shaffer RA, Maxwell-Williams K, Kao GL, Sartoris DJ, Brodine S. Dual-energy X-ray absorptiometry derived structural geometry for stress fracture prediction in male U.S. Marine Corps recruits. J Bone Miner Res. 1996;11(5):645-53.

37. Beck TJ, Ruff CB, Shaffer RA, Betsinger K, Trone DW, Brodine SK. Stress fracture in military recruits: gender differences in muscle and bone susceptibility factors. Bone. 2000;27(3):437-44. 